\title{
DNA mismatch repair in the context of chromatin
}

\author{
Yaping Huang ${ }^{*}$ (1) and Guo-Min Li(D)
}

\begin{abstract}
DNA mismatch repair (MMR) maintains replication fidelity by correcting mispaired nucleotides incorporated by DNA polymerases. Defects in MMR lead to cancers characterized by microsatellite instability. Recently, chromatin mechanisms that regulate MMR have been discovered, which sheds new light on MMR deficiency and its role in tumorigenesis. This review summarizes these chromatin-level mechanisms that regulate MMR and their implications for tumor development.
\end{abstract}

\section{Background}

DNA mismatch repair (MMR) maintains replication fidelity in a replication-coupled manner, primarily by correcting misincorporated nucleotides in the nascent DNA strand [1-4]. The typical MMR reaction in human cells comprises three major steps. First, the mismatch recognition protein MutS $\alpha$ (MSH2-MSH6 heterodimer) or MutS $\beta$ (MSH2-MSH3 heterodimer) recognizes the mismatch, which triggers concerted interactions with proliferating cell nuclear antigen (PCNA) and MutL $\alpha$ (MLH1-PMS2 heterodimer), leading to the recruitment of exonuclease 1 (EXO1) to a single-strand DNA break. Then, Exo1-catalyzed DNA excises the mispaired base from the nick up to and beyond the mismatch in a manner dependent on MutS $\alpha$ (or MutS $\beta$ ), MutL $\alpha$, and replication protein A (RPA). Finally, the DNA gap is filled by DNA polymerase $\delta$ in the presence of PCNA, RPA, and replication factor $\mathrm{C}$ (RFC), followed by DNA ligase I-catalyzed nick ligation.

In eukaryotes, DNA is wrapped around histone octamers, which, together with DNA, compose nucleosomes to form chromatin. Thus, all DNA metabolic reactions, including MMR, are precisely regulated by the structures of chromatin, particularly its component histone

*Correspondence: yaping.huang@utsouthwestern.edu Department of Radiation Oncology, University of Texas Southwestern Medical Center, Dallas, TX 75390, USA proteins and their modifications. Indeed, recent studies have indicated that trimethylation of histone $\mathrm{H} 3$ lysine 36 (H3K36me3) plays a role in MMR by recruiting MutS $\alpha$ to replicating chromatin [5]. In addition, chromatin assembly/remodeling factors also interact with MMR proteins to coordinate MMR and nucleosome formation $[6,7]$.

Loss-of-function mutations or promoter hyper-methylation of MMR genes, such as $M S H 2$ and $M L H 1$, increase susceptibility to cancers, including hereditary non-polyposis colorectal cancer (HNPCC), also called lynch syndrome $[1,2,4,8,9]$. The demonstration of H3K36me3 as an essential component for MMR in vivo [5] may provide new insights into MMR deficiency and cancer susceptibility in the chromatin context. H3K36me3 is a wellknown histone post-translational modification mark, and its cellular level is regulated by its trimethyltransferase SETD2 and lysine demethylase KDM4 [10]. Inappropriate expression of and/or defects in these histone writer and eraser genes probably influence cellular H3K36me3 levels, leading to the loss of MMR function.

Recent studies have also shown that mutations in histone $3(\mathrm{H} 3)$, particularly $\mathrm{H} 3 \mathrm{~K} 36 \mathrm{M} / \mathrm{I}$ and $\mathrm{H} 3 \mathrm{G} 34 \mathrm{~V} / \mathrm{R}$, which frequently occur in various tumors [11-14], affect H3K36me3 expression levels $[15,16]$. This review will focus on the most recent developments in the field concerning the regulation of MMR in the context of chromatin and its association with cancer susceptibility. Readers 
are also referred to several recent reviews in this area [17-20].

\section{Main text \\ H3K36me3 distribution dictates local mutation frequency} The biochemistry of MMR is essentially well established, but how the MMR system is regulated in the chromatin context is not fully understood. In 2013, Li et al. [5] identified H3K36me3 as an important MMR regulator in vivo by recruiting mismatch recognition protein MutS $\alpha$ to replicating chromatin through its physical interaction with the PWWP domain of human MutS $\alpha$. A recent chromatin immunoprecipitation followed by sequencing (ChIP-Seq) study [21] has illustrated the genome-wide distribution of H3K36me3 and MutS $\alpha$ in HeLa cells. Both $\mathrm{H} 3 \mathrm{~K} 36 \mathrm{me} 3$ and MutS $\alpha$ are unevenly distributed in chromatin [10, 21]. H3K36me3 and MutS $\alpha$ are more enriched in euchromatin, exons, and $3^{\prime}$ gene bodies than in heterochromatin, introns, and $5^{\prime}$ gene bodies, respectively. Consistent with MMR's genome maintenance role, the H3K36me3-MutS $\alpha$ distribution is inversely correlated with mutation frequencies in these genomic regions, as the mutation frequencies in euchromatin, exons, and $3^{\prime}$ gene bodies are much lower than in heterochromatin, introns, and $5^{\prime}$ gene bodies, respectively. In other words, the abundance of H3K36me3 and MutS $\alpha$ is inversely correlated to the local mutation frequency [21].

It is known that replication timing determines mutation frequency: early replicating genes exhibit lower mutation frequencies than late replicating genes. This phenomenon can be well explained by the distribution and enrichment of H3K36me3/MutS $\alpha$ in chromatin. Analyzing the relationship between replication timing, H3K36me3/MutS $\alpha$ enrichment, and mutation frequency revealed that early replicating chromatin regions are highly enriched for H3K36me3/MutS $\alpha$ and display a lower mutation frequency than late replicating regions, which contain fewer H3K36me3/MutS $\alpha$ signals [21]. These observations suggest that H3K36me3-mediated MMR ensures the replication accuracy of early replicating genes/chromatin, where actively transcribed genes are located. In addition to safeguarding actively transcribed genes during DNA replication, H3K36me3-mediated MMR also appears to protect these genes during transcription by directly or indirectly removing DNA lesions associated with transcription [21]. However, how H3K36me3 regulates MMR in different DNA transactions, e.g., replication and transcription, remains to be investigated.

It is worth noting that not all eukaryotic cells use H3K36me3 for MutSa recruitment. For example, despite that the abundance and distribution pattern of H3K36me3 in yeast genome are similar to those in human genome [22-24], yeast MSH6 does not have a
PWWP domain. Thus, yeast MutS $\alpha$ must be recruited by a histone mark different from H3K36me3, which is consistent with the report that set2 deficiency doesn't influence mutation frequency in yeast [25]. A recent study revealed that the PWWP domain of MSH6 in Capsella rubella interacts better with H3K4me3 than with H3K36me3 [26], suggesting that H3K4me3, rather than H3K36me3, is the preferred histone mark for loading MutS $\alpha$ to chromatin in plants. The difference in MutS $\alpha$ recruitment among different organisms may suggest an adapted evolution for MMR regulation in the chromatin context, although the canonical MMR function is highly conserved. Therefore, additional studies are needed to determine why different systems use different mechanisms for MutS $\alpha$ recruitment.

\section{Chromatin remodeling in MMR}

MMR occurs in the chromatin context, where nucleosome obstacles must be handled for efficient repair before the newly synthesized mismatch-containing DNA is packaged into nucleosomes. Thus, MMR needs to coordinate with chromatin remodeling factors and/or nucleosome assembly factors to repair misincorporated nucleotides (Fig. 1). CAF-1 is the most studied nucleosome assembly factor involved in MMR during replication. CAF-1 directly interacts with MMR components MutS $\alpha$ and PCNA, and MutS $\alpha$ inhibits CAF-1-mediated chromatin assembly [27]. Recent biochemical studies further indicate that MutS $\alpha$ inhibits CAF-1- and ASF1AH3-H4-dependent packaging of DNA mismatches into $(\mathrm{H} 3-\mathrm{H} 4)_{2}$ tetramers [28]. These studies support the idea that MMR occurs before the mismatch is packaged into nucleosomes. In addition, CAF-1- and ASF1A-H3-H4dependent nucleosome assembly quickly represses the unnecessary degradation of the discontinuous strand [7, 28]. Thus, nucleosome assembly is compatible with MMR during replication. In contrast to CAF-1, the chromatin remodeler Smarcad1 is recruited by MSH2 to mismatchcontaining DNA to exclude nucleosomes from the repair site [29]. It is interesting to note that, although MSH2, a subunit of MutS $\alpha$, can be recruited to chromatin via H3K36me3 [5], a recent study showed that ARID1A, a subunit of the chromatin remodeling complex SWI/ SNF, recruits MSH2 to the chromatin during replication through direct interaction [30]. Histone variants also play important roles in DNA transactions, in addition to nucleosome assembly and chromatin remodeling. It was reported that deposition of H2A.Z by chromatin remodeling enzyme SWR-C stimulates Exo1 activity and enhances MMR during replication, although the mechanism is unclear [31]. Thus, it is worth examining how the chromatin remodeling machinery and histone variants modulate MMR in future studies. 


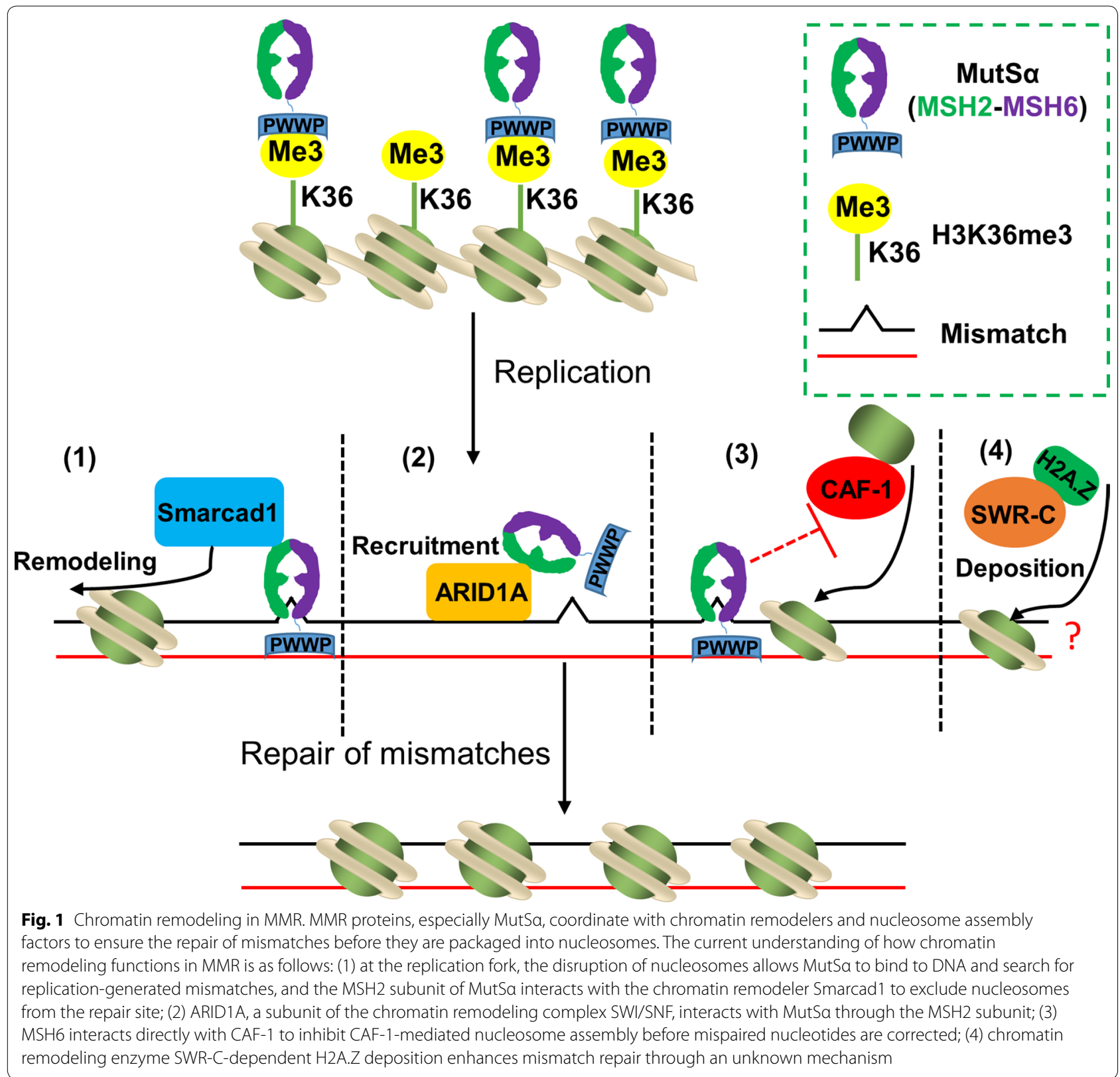

\section{Histone mutations affecting H3K36me3 levels impair} mismatch repair

In the past few years, driver mutation hotspots targeted to histone H3.3-including H3.3K27M, H3.3G34V/R and H3.3K $36 \mathrm{M} / \mathrm{I}$ amino acid substitutions-have been identified in various tumors (Table 1) [11-14, 32, 33]. The H3.3K27M and H3.3G34V/R mutation signatures are specific to pediatric glioblastomas (GBMs), making these mutation signatures new biomarkers for GBM subtyping and diagnosis. Further studies have demonstrated that pediatric GBMs can be further subtyped by $\mathrm{K} 27 \mathrm{M}$ and $\mathrm{G} 34 \mathrm{~V} / \mathrm{R}$ mutations [14, 34]. Functional studies have demonstrated that $\mathrm{H} 3 \mathrm{~K} 27 \mathrm{M}$ is a gainof-function mutation that results in genome-wide depletion of H3K27me3 and disrupts gene expression patterns, which promotes tumorigenesis [11, 35]. $\mathrm{H} 3 \mathrm{~K} 36 \mathrm{M} / \mathrm{I}$ substitution has been reported to block the methyltransferase from methylating H3K36, which reprograms the genome-wide H3K36 methylation pattern $[12,32]$. The H3K36me3 level is dramatically lower in cells with $\mathrm{H} 3 \mathrm{~K} 36 \mathrm{M} / \mathrm{I}$ mutations [12, 33], which impairs the H3K36me3-dependent pathways, including MMR. However, until recently, the function of G34V/R remained obscure, although it was postulated that these 
Table 1 Factors modulating H3K36me3 and their susceptibility to cancers

\begin{tabular}{|c|c|c|}
\hline Function & Enzyme & Related cancers \\
\hline \multirow[t]{9}{*}{ Methyltransferase } & ASH1L & Sinonasal neuroendocrine tumors, lung cancer, prostate cancer \\
\hline & NSD1 & Acute myeloid leukemia, prostate, neuroblastoma, breast, lung, glioma \\
\hline & NSD2 & Multiple myeloma, acute lymphocytic leukemia, prostate \\
\hline & NSD3 & Acute myeloid leukemia, breast cancer \\
\hline & PRDM9 & Acute lymphocytic leukemia \\
\hline & SETD2 & $\begin{array}{l}\text { Renal clear cell carcinoma, lymphoblastic leukemia, breast cancer, prostate cancer, } \\
\text { lung cancer, glioma, thymic carcinoma, acute myeloid leukemia }\end{array}$ \\
\hline & SETD3 & Renal cell carcinoma, B-cell lymphomas \\
\hline & SETMAR & Acute myeloid leukemia, breast cancer \\
\hline & SMYD2 & Renal cell, acute myeloid leukemia, chronic lymphocytic leukemia, breast cancer \\
\hline \multirow[t]{6}{*}{ Demethylase } & KDM2A & Gastric, breast, lung \\
\hline & KDM2B & Pancreatic, hematologic \\
\hline & KDM4A & Breast, prostate \\
\hline & KDM4B & Breast, prostate, colon, gastric, lung, melanoma \\
\hline & KDM4C & Breast, lung, prostate, melanoma, lymphoma \\
\hline & N066 & Renal cell, colorectal \\
\hline \multirow[t]{2}{*}{ Histone mutation } & $\mathrm{H} 3 \mathrm{~K} 36 \mathrm{M} / \mathrm{I}$ & Glioblastoma, head and neck squamous cell carcinoma, chondroblastomas, sarcoma \\
\hline & $\mathrm{H} 3 \mathrm{G} 34 \mathrm{~V} / \mathrm{R} / \mathrm{D}$ & Glioblastoma, glioma, chondroblastomas, sarcoma, colon \\
\hline \multirow[t]{4}{*}{ Other factors } & ASF1 & NA \\
\hline & CTK1 & NA \\
\hline & $\mathrm{IDH} 1$ & Acute myeloid leukemia, glioma \\
\hline & SPT6 & Skin, bladder, colorectal \\
\hline
\end{tabular}

mutations drive tumorigenesis by upregulating MYCN [34].

Our group recently found that $\mathrm{H} 3 \mathrm{G} 34 \mathrm{~V} / \mathrm{R}$ mutations also block H3K36 methylation by methyltransferases and result in MMR deficiency [16]. This is because these mutations inhibit H3K36's interactions with SETD2 and MutS $\alpha$. Thus, cells carrying these mutations display MMR-deficient phenotypes, including microsatellite instability (MSI) and elevated mutation frequency at the HPRT locus (Fig. 2). The co-crystal structure revealed that the SET domain of the SETD2 protein adopts a closed conformation for the H3K36 peptide [15], and G34V/R mutations significantly increase the space blockage resulting from the big side chain of $V / R$ residues, which impairs H3K36 methylation [16]. Similarly, Voon et al. [36] revealed that G34V/R mutations inhibit H3K36 demethylase KDM4 to modulate the H3K36me3 pattern in pediatric glioblastoma. Therefore, both $\mathrm{K} 36 \mathrm{M} / \mathrm{I}$ and G34V/R mutations inhibit H3K36 methylation and are promising biomarkers for MMR-deficient cancers.

It is worth noting that these histone mutations frequently target the H3.3 variant but not the $\mathrm{H} 3.1$ or $\mathrm{H} 3.2$ variants in GBM and gliomas [13, 14, 37]. Glioma cells are terminally-differentiated cells with limited cell division. More importantly, H3.3 are preferentially incorporated into nucleosomes during transcription by $\mathrm{H} 3.3$-specific chaperons [38]. The question is how these mutations could target MMR, which is commonly regarded as a process coupled to DNA replication in dividing cells. One possible answer is that H3K36me3-mediated MMR also functions during transcription $[17,21]$. Thus, it is feasible that H3.3K36me3 is important for MMR to maintain genome stability during transcription in the non-dividing neuron cells (Fig. 2a). Therefore, these findings largely broaden what the histone code regulates in MMR and tumorigenesis.

\section{Chromatin remodeling in MMR-mediated cytotoxic response}

In addition to repairing mismatched DNA, MMR also functions in non-canonical ways to promote cytotoxic responses to cell stresses, such as $O^{6}$-methylguanine $\left(O^{6}-\mathrm{mG}\right)$ - and oxidation-induced cell apoptosis [4, 39]. MMR proteins recognize and incise the $O^{6}-\mathrm{mG}-\mathrm{T}$ mispair, but cannot correct it, which leads to a futile repair cycle and, finally, apoptosis. Similar to its role in replication-coupled repair, CAF-1 also suppresses MMR activity in response to DNA methylating drugs by packaging DNA that contains $O^{6}$-mG-T mispairs into nucleosomes to prevent it from degrading [6], which may lead to cell resistance to methylating agents, such as methyl-nitronitrosoguanidine (MNNG). Similarly, Smarcad1 may 


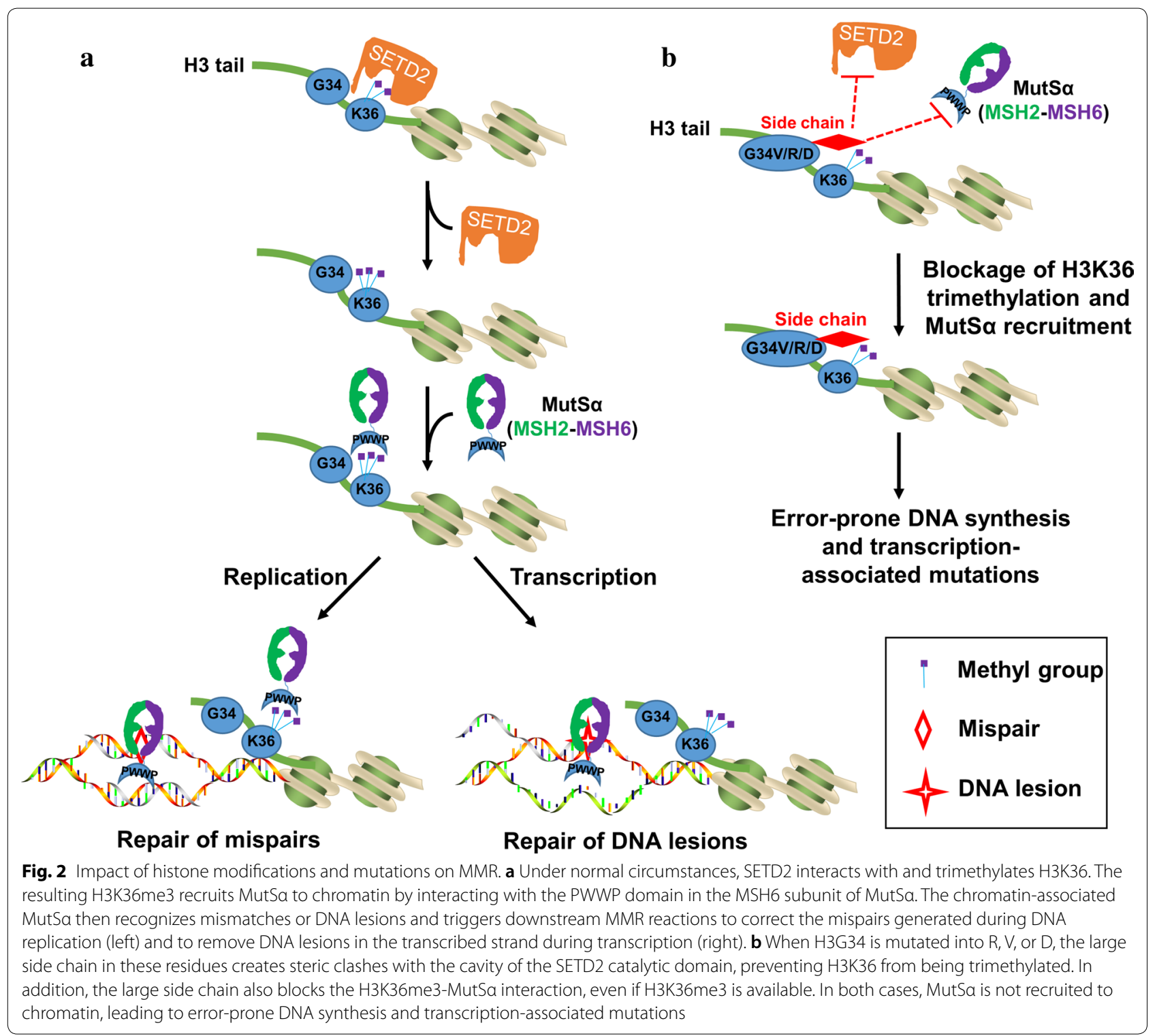

sensitize cells to methylating agents by promoting MMRmediated cytotoxic responses. Since MMR is important for cancer cell killing mediated by methylating drugs, and since chromatin regulation of MMR is important in vivo, future studies are required to investigate the detailed functions of chromatin remodeling factors in MMR and their potential as targets for sensitizing cancer cells to methylating drugs.

\section{Impact of histone methyltransferases and demethylases on H3K36me3 and MMR}

It is well-known that MMR deficiency causes cancers characterized by MSI $[2,4,40]$. However, not all MSIpositive cancers display MMR gene defects [41, 42]. The underlying mechanism for these MSI cancers was not clear until H3K36me3 was identified as an essential regulator of MMR in vivo [5]. Thus, defects in H3K36me3 are considered a promising biomarker for MSI-positive cancers. Recent studies have shown that MMR deficiency or MSI benefits from immune checkpoint inhibitor therapy for patients with many types of cancer [43, 44]. Thus, a comprehensive list of factors that influence H3K36me3 levels should be examined before considering patients with MSI-positive tumors for immunotherapy.

SETD2 is the major methyltransferase that converts H3K36me2 to H3K36me3 in mammalian cells [10]. However, H3K36me3 metabolism is tightly regulated by multiple histone methyltransferases, demethylases 
and other factors. These methyltransferases include the SET domain-containing proteins and the DOT1-like proteins, i.e., SETD2, SETD3, SETMAR, NSD1, NSD2, NSD3, ASH1L, and SMYD2 [10, 45]. Recently, another methyltransferase, PRDM9, was also reported to trimethylate H3K36 in certain contexts [46]. In contrast to histone methyltransferases, histone demethylases, such as KDM2A, KDM2B, KDM4A, KDM4B, KDM4C, and NO66, can remove methyl groups from H3K36 [47]. In addition, histone chaperons or deposition factors are also important for properly assembling different $\mathrm{H} 3$ variants into nucleosomes and establishing corresponding methylation patterns $[48,49]$. As an important epigenetic regulator, H3K36 methylation modulates many cellular/ chromatin functions, and defects in H3K36me3 metabolism are susceptible to causing human diseases [45, 47], including cancers.

Frequent mutations in the aforementioned methyltransferase genes have been identified in different cancer types (Table 1). For example, the SETD2 loss-of-function mutations were found in renal carcinoma [50,51], lung cancer [52, 53], gastrointestinal cancer [54], and hematologic malignancies $[55,56]$, indicating that SETD2 is a tumor suppressor. Similarly, other methyltransferases have also been reported susceptible to causing different types of cancer (Table 1). However, the outcome of these mutations targeting histone writer enzymes, especially the NSD family, is complex, as they lead either to losses of function or to gains of function [10]. Since H3K36me3 is essential for MMR in vivo, H3K36me3 depletion resulting from the loss of H3K36 mono-, di- and tri-methyltransferases will result in MMR deficiency and genome instability. It is also possible that these enzymes interact directly with MMR proteins to regulate MMR function in vivo. Therefore, further studies are required to demonstrate whether and how mutations of these histone methyltransferases impair H3K36 metabolism and/or MMR to promote cancer development.

In addition to histone methyltransferase mutations, depleting H3K36me3 by overexpressing H3K36me2/3 demethylases, e.g., KDM4A-C, also disrupts MSH6 chromatin localization and induces MMR-deficient phenotypes [57]. Consistent with the idea that histone demethylases function against SETD2 in MMR, mutation-led upregulation of these histone demethylases, resulting from gene fusion, amplification or overactivation [58-60], is also susceptible to causing various types of cancer (Table 1). It is likely that overexpression of these genes erases the level of H3K36 methylation in cells, which disrupts the H3K36me3-mediated MMR pathway and causes genome instability. Thus, these genes are also promising biomarkers for H3K36me3-MMR deficient cancers.

\section{Other $\mathrm{H} 3 \mathrm{~K} 36$ methylation regulators}

Besides histone methyltransferases/demethylases and histone $\mathrm{H} 3$ mutations, other factors are also involved in regulating normal H3K36 methylation patterns in cells (Table 1). For example, IDH1 is frequently mutated in gliomas, and its metabolic product 2-hydroxyglutarate inhibits lysine demethylase activity [13, 61]. The histone chaperons ASF1, CTK1 and SPT6 have been demonstrated to modulate transcription-associated SETD2-mediated H3K36 methylation [48, 49]. Thus, these factors may also modulate H3K36me3-mediated MMR in vivo. Future studies are required to understand the mechanisms by which these factors regulate MMR, which will provide a more accurate and complete understanding of non-classical mechanisms by which abnormal epigenetic factors cause MMR defects and cancer susceptibility, as well as new targets or strategies for preventing and treating tumors.

\section{Conclusion}

MMR is a replication-coupled reaction. Even though the reaction has been reconstituted [62-64], it is not yet fully understood how MMR and DNA replication are coordinated at the replication fork and how chromatin structures modulate the coupled MMR and replication processes. Identifying the involvement of histone mark H3K36me3 and chromatin remodeling factors in regulating MMR in vivo only marks the beginning of this exciting new area. We believe that future studies will identify other histone modifications and/or chromatin factors, in addition to H3K36me3 and the reported chromatin remodelers, that regulate the MMR system. Given the importance of MMR in cancer etiology and therapy $[2,41,65]$, it is expected that these new factors will greatly advance cancer diagnosis and treatment.

\section{Acknowledgements \\ The authors thank Dr. Jonathan Feinberg for editing. G.-M.L. holds the Reece A Overcash, Jr. Distinguished Chair for Research on Colon Cancer.}

\section{Authors' contributions}

YH wrote the draft. GML supervised the project and revised the manuscript. Both authors read and approved the final manuscript.

\section{Funding}

This work is supported in part by grants from the National Institutes of Health (GM112702) and the Cancer Prevention \& Research Institute of Texas (RR160101) to G.-M.L.

\section{Availability of data and materials} Not applicable.

\section{Ethics approval and consent to participate}

Not applicable.

Consent for publication

Not applicable. 


\section{Competing interests}

The authors declare that they have no competing interests.

Received: 11 December 2019 Accepted: 24 January 2020

Published online: 03 February 2020

\section{References}

1. Kolodner R. Biochemistry and genetics of eukaryotic mismatch repair Genes Dev. 1996;10(12):1433-42.

2. Modrich P, Lahue R. Mismatch repair in replication fidelity, genetic recombination, and cancer biology. Annu Rev Biochem. 1996;65:101-33.

3. Kunkel TA, Erie DA. Eukaryotic mismatch repair in relation to DNA replication. Annu Rev Genet. 2015:49:291-313.

4. Li GM. Mechanisms and functions of DNA mismatch repair. Cell Res. 2008;18(1):85-98.

5. Li F, et al. The histone mark H3K36me3 regulates human DNA mismatch repair through its interaction with MutSalpha. Cell. 2013;153(3):590-600.

6. Kadyrova LY, Dahal BK, Kadyrov FA. The major replicative histone chaperone CAF-1 suppresses the activity of the DNA mismatch repair system in the cytotoxic response to a DNA-methylating agent. J Biol Chem. 2016;291(53):27298-312.

7. Kadyrova LY, Blanko ER, Kadyrov FA. CAF-I-dependent control of degradation of the discontinuous strands during mismatch repair. Proc Natl Acad Sci USA. 2011;108(7):2753-8.

8. Parsons R, et al. Hypermutability and mismatch repair deficiency in RER + tumor cells. Cell. 1993;75(6):1227-36.

9. Kane MF, et al. Methylation of the hMLH1 promoter correlates with lack of expression of hMLH1 in sporadic colon tumors and mismatch repairdefective human tumor cell lines. Cancer Res. 1997;57(5):808-11.

10. Wagner EJ, Carpenter PB. Understanding the language of Lys36 methylation at histone H3. Nat Rev Mol Cell Biol. 2012;13(2):115-26.

11. Lewis PW, et al. Inhibition of PRC2 activity by a gain-of-function H3 mutation found in pediatric glioblastoma. Science. 2013;340(6134):857-61.

12. Fang $\mathrm{D}$, Gan $\mathrm{H}$, Lee JH, et al. The histone $\mathrm{H3} 3.3 \mathrm{~K} 36 \mathrm{M}$ mutation reprograms the epigenome of chondroblastomas. Science. 2016:352(6291):1344-8.

13. Sturm D, et al. Hotspot mutations in $\mathrm{H} 3 \mathrm{~F} 3 \mathrm{~A}$ and IDH1 define distinct epigenetic and biological subgroups of glioblastoma. Cancer Cell. 2012:22(4):425-37.

14. Schwartzentruber J, et al. Driver mutations in histone $\mathrm{H} 3.3$ and chromatin remodelling genes in paediatric glioblastoma. Nature. 2012;482(7384):226-31.

15. Yang $\mathrm{S}$, et al. Molecular basis for oncohistone $\mathrm{H} 3$ recognition by SETD2 methyltransferase. Genes Dev. 2016;30(14):1611-6.

16. Fang J, et al. Cancer-driving H3G34V/R/D mutations block H3K36 methylation and H3K36me3-MutSa interaction. Proc Natl Acad Sci USA 2018;115(38):9598-603.

17. Huang Y, Li GM. DNA mismatch repair preferentially safeguards actively transcribed genes. DNA Repair. 2018;71:82-6.

18. Li F, et al. Regulation of mismatch repair by histone code and posttranslational modifications in eukaryotic cells. DNA Repair. 2016;38:68-74.

19. Li GM. New insights and challenges in mismatch repair: getting over the chromatin hurdle. DNA Repair. 2014;19:48-54.

20. Goellner E. Chromatin remodeling and mismatch repair: access and excision. DNA Repair. 2019;85:102733.

21. Huang Y, Gu L, Li GM. H3K36me3-mediated mismatch repair preferentially protects actively transcribed genes from mutation. J Biol Chem. 2018;293(20):7811-23.

22. Sadeh $\mathrm{R}$, et al. Elucidating combinatorial chromatin states at single-nucleosome resolution. Mol Cell. 2016;63(6):1080-8.

23. Wilhelm BT, et al. Differential patterns of intronic and exonic DNA regions with respect to RNA polymerase II occupancy, nucleosome density and H3K36me3 marking in fission yeast. Genome Biol. 2011;12(8):R82.

24. Janevska S, et al. Elucidation of the two H3K36me3 histone methyltransferases Set2 and Ash1 in Fusarium fujikuroi unravels their different chromosomal targets and a major impact of Ash1 on genome stability. Genetics. 2018;208(1):153-71.
25. Sun L, et al. Preferential protection of genetic fidelity within open chromatin by the mismatch repair machinery. J Biol Chem. 2016:291(34):17692-705.

26. Zhao $\mathrm{S}$, et al. Kinetic and high-throughput profiling of epigenetic interactions by 3D-carbene chip-based surface plasmon resonance imaging technology. Proc Natl Acad Sci USA. 2017;114(35):E7245-54.

27. Schöpf B, et al. Interplay between mismatch repair and chromatin assembly. Proc Natl Acad Sci USA. 2012;109(6):1895-900.

28. Blanko ER, Kadyrova LY, Kadyrov FA. DNA Mismatch repair interacts with CAF-1- and ASF1A-H3-H4-dependent histone (H3-H4)2 tetramer deposition. J Biol Chem. 2016;291(17):9203-17.

29. Terui $R$, et al. Nucleosomes around a mismatched base pair are excluded via an Msh2-dependent reaction with the aid of SNF2 family ATPase Smarcad1. Genes Dev. 2018;32(11-12):806-21.

30. Shen J, et al. ARID1A deficiency promotes mutability and potentiates therapeutic antitumor immunity unleashed by immune checkpoint blockade. Nat Med. 2018;24(5):556-62

31. Van $\mathrm{C}$, et al. Deposition of histone $\mathrm{H} 2 \mathrm{~A} . \mathrm{Z}$ by the SWR-C remodeling enzyme prevents genome instability. DNA Repair. 2015;25:9-14.

32. Lu C, Jain SU, Hoelper D, et al. Histone H3K36 mutations promote sarcomagenesis through altered histone methylation landscape. Science. 2016;352(6287):844-9.

33. Papillon-Cavanagh S, Lu C, Gayden T, et al. Impaired H3K36 methylation defines a subset of head and neck squamous cell carcinomas. Nat Genet. 2017:49(2):180-5.

34. Bjerke L, et al. Histone H3.3 mutations drive pediatric glioblastoma through upregulation of MYCN. Cancer Discov. 2013;3(5):512-9.

35. Bender S, et al. Reduced H3K27me3 and DNA hypomethylation are major drivers of gene expression in K27M mutant pediatric high-grade gliomas. Cancer Cell. 2013;24(5):660-72.

36. Voon HPJ, et al. Inhibition of a K9/K36 demethylase by an H3.3 point mutation found in paediatric glioblastoma. Nat Commun. 2018;9(1):3142-51.

37. Yuen BT, Knoepfler PS. Histone H3.3 mutations: a variant path to cancer. Cancer Cell. 2013;24(5):567-74.

38. Ahmad K, Henikoff S. The histone variant H3.3 marks active chromatin by replication-independent nucleosome assembly. Mol Cell. 2002:9(6):1191-200.

39. Crouse GF. Non-canonical actions of mismatch repair. DNA Repair 2016:38:102-9.

40. Kinzler KW, Vogelstein B. Lessons from hereditary colorectal cancer. Cell. 1996:87(2):159-70.

41. Peltomaki P. Role of DNA mismatch repair defects in the pathogenesis of human cancer. J Clin Oncol. 2003:21:1174-9.

42. Gu L, et al. Mismatch repair deficiency in hematological malignancies with microsatellite instability. Oncogene. 2002;21:5758-64

43. Le DT, et al. PD-1 blockade in tumors with mismatch-repair deficiency. $N$ Engl J Med. 2015;372(26):2509-20.

44. Dudley JC, et al. Microsatellite instability as a biomarker for PD-1 blockade. Clin Cancer Res. 2016;22(4):813-20

45. Albert M, Helin K. Histone methyltransferases in cancer. Semin Cell Dev Biol. 2010;21:209-20.

46. Powers NR, Parvanov E, Baker CL, Walker M, Petkov PM, Paigen K. The meiotic recombination activator PRDM9 trimethylates both $\mathrm{H} 3 \mathrm{~K} 36$ and $\mathrm{H} 3 \mathrm{~K} 4$ at recombination hotspots in vivo. PLoS Genet. 2016;12(6):e1006146.

47. Greer EL, Shi Y. Histone methylation: a dynamic mark in health, disease and inheritance. Nat Rev Genet. 2012;13:343-57.

48. Yoh SM, Lucas J, Jones KA. The Iws 1:Spt6:CTD complex controls cotranscriptional mRNA biosynthesis and HYPB/Setd2-mediated histone H3K36 methylation. Genes Dev. 2008;22(24):3422-34.

49. Youdell ML, Kizer K, Kisseleva-Romanova E, Fuchs SM, Duro E, Strahl BD, Mellor J. Roles for Ctk1 and Spt6 in regulating the different methylation states of histone H3 lysine 36. Mol Cell Biol. 2008;28(16):4915-26.

50. Dalgliesh GL, Furge K, Greenman C, Chen L, Bignell G, Butler A, et al. Systematic sequencing of renal carcinoma reveals inactivation of histone modifying genes. Nature. 2010:463:360-3.

51. Duns G, van den Berg E, van Duivenbode I, Osinga J, Hollema H, Hofstra RM, et al. Histone methyltransferase gene SETD2 is a novel tumor suppressor gene in clear cell renal cell carcinoma. Cancer Res. 2010;70:4287-91. 
52. Govindan R, Ding L, Griffith M, Subramanian J, Dees N, Walker KL, et al. Genomic landscape of non-small cell lung cancer in smokers and neversmokers. Cell. 2012;150:1121-34.

53. Imielinski M, Berger AH, Hammerman PS, Hernandez B, Pugh TJ, Hodis E, et al. Mapping the hallmarks of lung adenocarcinoma with massively parallel sequencing. Cell. 2012;150:1107-20.

54. Huang KK, McPherson J, Tay ST, et al. SETD2 histone modifier loss in aggressive GI stromal tumours. Gut. 2015;65(12):1960-72.

55. Zhu X, He F, Zeng H, et al. Identification of functional cooperative mutations of SETD2 in human acute leukemia. Nat Genet. 2014;46(3):287-93.

56. Zhang J, Ding L, Holmfeldt L, Wu G, Heatley SL, Payne-Turner D, et al. The genetic basis of early T-cell precursor acute lymphoblastic leukaemia. Nature. 2012:481:157-63.

57. Awwad SW, Ayoub N. Overexpression of KDM4 lysine demethylases disrupts the integrity of the DNA mismatch repair pathway. Biol Open. 2015;4(4):498-504.

58. Young LC, Hendzel M. The oncogenic potential of Jumonji D2 (JMJD2/ KDM4) histone demethylase overexpression. Biochem Cell Biol. 2013;91(6):369-77.

59. Berry WL, Janknecht R. KDM4/JMJD2 histone demethylases: epigenetic regulators in cancer cells. Cancer Res. 2013;73(10):2936-42.
60. Nishizawa Y, Nishida N, Konno M, Kawamoto K, Asai A, Koseki J, Takahashi H, Haraguchi N, Nishimura J, Hata T, Matsuda C, Mizushima T, Satoh T, Doki Y, Mori M, Ishii H. Clinical significance of histone demethylase NO66 in invasive colorectal cancer. Ann Surg Oncol. 2017;24(3):841-9.

61. Fontebasso AM, Schwartzentruber J, Khuong-Quang DA, et al. Mutations in SETD2 and genes affecting histone H3K36 methylation target hemispheric high-grade gliomas. Acta Neuropathol. 2013;125(5):659-69.

62. Zhang Y, et al. Reconstitution of $5^{\prime}$-directed human mismatch repair in a purified system. Cell. 2005;122(5):693-705.

63. Constantin $\mathrm{N}$, et al. Human mismatch repair: reconstitution of a nickdirected bidirectional reaction. J Biol Chem. 2005;280(48):39752-61.

64. Bowen N, et al. Reconstitution of long and short patch mismatch repair reactions using Saccharomyces cerevisiae proteins. Proc Natl Acad Sci USA. 2013;110(46):18472-7.

65. Baretti M, Le DT. DNA mismatch repair in cancer. Pharmacol Ther. 2018;189:45-62.

\section{Publisher's Note}

Springer Nature remains neutral with regard to jurisdictional claims in published maps and institutional affiliations.
Ready to submit your research? Choose BMC and benefit from:

- fast, convenient online submission

- thorough peer review by experienced researchers in your field

- rapid publication on acceptance

- support for research data, including large and complex data types

- gold Open Access which fosters wider collaboration and increased citations

- maximum visibility for your research: over $100 \mathrm{M}$ website views per year

At BMC, research is always in progress.

Learn more biomedcentral.com/submissions 